Author Index to Volume 36 - 2009

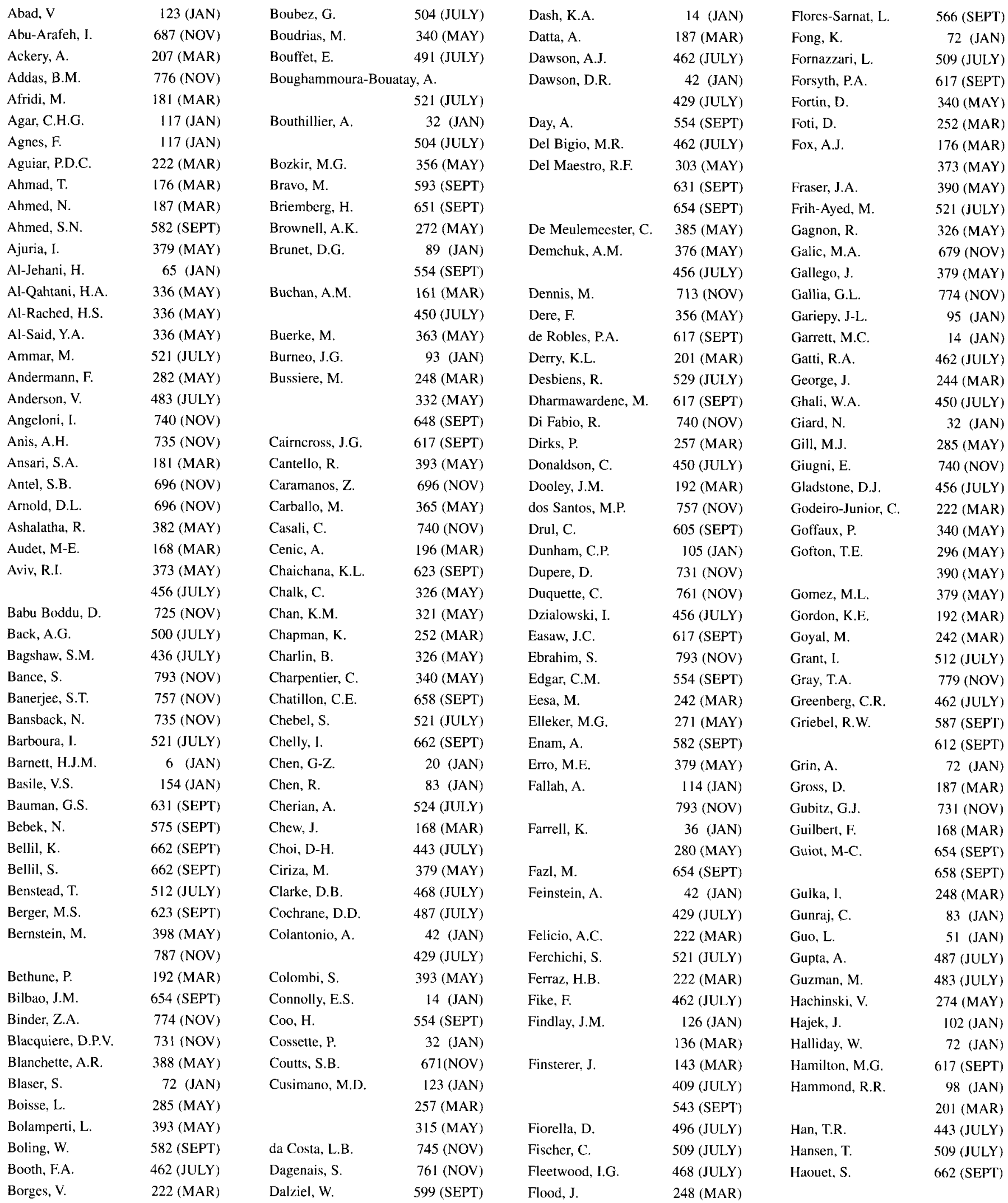


Author Index to Volume 36 - 2009

\begin{tabular}{|c|c|c|c|c|c|c|c|}
\hline \multirow[t]{2}{*}{ Hawkins, $\mathrm{C}$. } & 257 (MAR) & Karanjia, R. & $89(\mathrm{JAN})$ & Lena, T.S. & 385 (MAY) & Mohammed, S. & $123(\mathrm{JAN})$ \\
\hline & 491 (JULY) & Kaszas, $\mathrm{K}$. & 234 (MAR) & Lescrauwaet, B. & 599 (SEPT) & Moien-Afshari, $F$ & 587 (SEPT) \\
\hline Harrati, L-N. & 244 (MAR) & Kaul, S. & 725 (NOV) & Letoha, $\mathrm{T}$. & 234 (MAR) & Monaco, $\mathrm{F}$. & 393 (MAY) \\
\hline Hedden, D. & 47 (JAN) & Kaya, M. & 356 (MAY) & Leung, A. & 648 (SEPT) & Moore, F.G.A. & 769 (NOV) \\
\hline Heidari, M.M. & $26(\mathrm{JAN})$ & Kazitani, D. & 326 (MAY) & $\mathrm{Li}, \mathrm{L}$. & 349 (MAY) & Moriarity, J.L. & 774 (NOV) \\
\hline Henriques, L. & 491 (JULY) & Kehir, N. & 662 (SEPT) & Lim, K.S. & $60(\mathrm{JAN})$ & Moriones, I. & 379 (MAY) \\
\hline \multirow[t]{2}{*}{ Heran, M.K.S. } & 252 (MAR) & Kecskes, M. & 234 (MAR) & Lim, T.S. & $60(\mathrm{JAN})$ & Morrow, S.A. & 213 (MAR) \\
\hline & 487 (JULY) & Keegan, B.M. & $562(\mathrm{SEPT})$ & Limaiem, F. & 662 (SEPT) & Moskau, S. & 789 (NOV) \\
\hline \multicolumn{2}{|c|}{ Hernandez-Ronquillo, L. } & Keeler, A. & 509 (JULY) & Linnebank, M. & 789 (NOV) & Moumdjian, R. & 504 (JULY) \\
\hline & 587 (SEPT) & Keene, D. & 707 (NOV) & Lisonkova, S. & $36(J A N)$ & Moussa, R. & 507 (JULY) \\
\hline Hershey, A.D. & 687 (NOV) & Keezer, M.R. & 303 (MAY) & Lownie, S.P. & 248 (MAR) & Mula, M. & 393 (MAY) \\
\hline Hew, Y.C. & $60(\mathrm{JAN})$ & Keith-Rokosh, J.A. & 201 (MAR) & & $332(\mathrm{MAY})$ & Mulroy, L.A. & 468 (JULY) \\
\hline \multirow[t]{2}{*}{ Hill, M.D. } & 161 (MAR) & Kellner, C.P. & $14(\mathrm{JAN})$ & Lowry, N. & 587 (SEPT) & Nadeau, Y. & 529 (JULY) \\
\hline & $450($ JULY) & Kelly, K.D. & $605(\mathrm{SEPT})$ & Lubarsky, S. & 326 (MAY) & Nafissi, S. & $26(\mathrm{JAN})$ \\
\hline Hodaie, M. & 244 (MAR) & Kelly, M.E. & 496 (JULY) & Luis, G.A.J. & 117 (JAN) & Narayansingh, M.J. & 262 (MAR) \\
\hline Hogan, M.J. & 757 (NOV) & & 612 (SEPT) & Luis, S.H.J. & $117(\mathrm{JAN})$ & Nayak, D. & 524 (JULY) \\
\hline Hopman, W.M. & $554(\mathrm{SEPT})$ & Kenny, B. & 78 (IAN) & Lum, C. & 480 (JULY) & Neeraja, M. & 725 (NOV) \\
\hline Hopyan, J.J. & 456 (JULY) & Keystone, J. & $102(\mathrm{JAN})$ & MacDonald, E.A. & $783(\mathrm{NOV})$ & Nicholle, M.N. & 201 (MAR) \\
\hline Horvath, E. & $123(\mathrm{JAN})$ & Khatami, M. & $26(\mathrm{JAN})$ & Machado, C. & $365(\mathrm{MAY})$ & Nguyen, D.K. & 32 (JAN) \\
\hline Hosseinkhani, S. & $26(\mathrm{JAN})$ & Khealani, B.A. & 181 (MAR) & Maeda, M. & 349 (MAY) & Nguyen-Huynh, M.N. & 403 (JULY) \\
\hline Hoube, J.S. & 36 (JAN) & Khu, K.J. & 787 (NOV) & Magliocco, A.M. & 617 (SEPT) & Noel de Tilly, L. & 176 (MAR) \\
\hline Houshmand, M. & 26 (JAN) & Khuong, $\mathrm{H}$. & $95(\mathrm{JAN})$ & Majl, L. & 244 (MAR) & & 779 (NOV) \\
\hline Hsu, F.D. & 776 (NOV) & Kikutani, $\mathrm{H}$. & 349 (MAY) & Mann, C. & 582 (SEPT) & Norris, J.W. & 274 (MAY) \\
\hline Huange, S. & 98 (JAN) & Kim, D-Y. & 443 (JULY) & Mann, G.S. & 487 (JULY) & Norton, J. & 47 (JAN) \\
\hline Hunter, G. & 518 (JULY) & Kim, G.H. & 14 (JAN) & Manns, B.J. & $450(\mathrm{JULY})$ & O'Connell, C.M. & 468 (JULY) \\
\hline Huntsman, R.J. & $105(\mathrm{JAN})$ & Kim, J.Y. & 515 (JULY) & Marotta, T.R. & 745 (NOV) & Odier, C. & $32(\mathrm{JAN})$ \\
\hline Hwang, S.H. & 515 (JULY) & King, J. & 257 (MAR) & Masiowski, P. & $370(\mathrm{MAY})$ & Oh, J. & 654 (SEPT) \\
\hline Hyson, C. & $98(\mathrm{JAN})$ & Kirk, A. & 531 (JULY) & Massoud, F. & 599 (SEPT) & Olah, Z. & 234 (MAR) \\
\hline Hyson, H.C. & 390 (MAY) & Kirton, A. & $83(\mathrm{JAN})$ & & 673 (NOV) & Olaz, $\mathrm{F}$. & 379 (MAY) \\
\hline Imran, S.A. & 468 (JULY) & Knafo, $\mathrm{H}$. & 78 (JAN) & Mathieu, D. & 78 (JAN) & Olivier, A. & 65 (JAN) \\
\hline Iso, $\mathrm{H}$ & 349 (MAY) & Kohsaka, A. & 349 (MAY) & & 340 (MAY) & & 593 (SEPT) \\
\hline Ivan, C.M. & $117(\mathrm{JAN})$ & Komotar, R.J. & $14(\mathrm{JAN})$ & McBride, E.V. & 554 (SEPT) & Orders, $\mathrm{S}$. & 761 (NOV) \\
\hline Jacka, M.J. & 436 (JULY) & Korein, J. & $365(\mathrm{MAY})$ & McCombe, J.A. & 262 (MAR) & Otten, M.L. & 14 (JAN) \\
\hline Jan, M.M.S. & $336(\mathrm{MAY})$ & Kornhuber, $\mathrm{M}$. & 363 (MAY) & McCullough-Hicks, & E. 14 (JAN) & Papp, A. & 234 (MAR) \\
\hline Javidan, M. & $677((\mathrm{NOV})$ & Kosior, J.C. & 456 (JULY) & McDermott, M.W. & 623 (SEPT) & Park, H-W. & 443 (JULY) \\
\hline Jawaid, A. & 388 (MAY) & Kovacs, K. & $123(\mathrm{JAN})$ & McDonald, P. & 791 (NOV) & Park, S-H. & 443 (JULY) \\
\hline Jemel, $\mathrm{H}$. & $662(\mathrm{SEPT})$ & Kremenchutzky, M. & 213 (MAR) & McDonald, W. & 631 (SEPT) & Parney, I.F. & 617 (SEPT) \\
\hline Jenkins, M.E. & 93 (JAN) & Kumanogoh, A. & 349 (MAY) & McEvilly, R. & 373 (MAY) & Parsa, A.T. & 623 (SEPT) \\
\hline Jette, N. & $2(\mathrm{JAN})$ & Kumar, $\mathrm{H}$. & 370 (MAY) & McInnes, M. & $72(\mathrm{JAN})$ & Pavlov, A. & 554 (SEPT) \\
\hline Jhamandas, J.H. & $262(\mathrm{MAR})$ & Kurt, S. & 575 (SEPT) & McIntyre, J.B. & 617 (SEPT) & Pecze, L. & 234 (MAR) \\
\hline Jhas, S. & 491 (JULY) & Kuznetsov, Y.E. & 696 (NOV) & McLeod, D. & 731 (NOV) & Pelsoczi, P. & 234 (MAR) \\
\hline Jiang, $Y$. & $51 \quad(\mathrm{JAN})$ & Kwon, K.H. & 515 (JULY) & Mehrotra, N. & 751 (NOV) & Pelz, D.M. & 93 (JAN) \\
\hline \multirow[t]{2}{*}{ Jog, M.S. } & 296 (MAY) & Kwon, S-B. & 515 (JULY) & Mekni, A. & 662 (SEPT) & & 248 (MAR) \\
\hline & $370(\mathrm{MAY})$ & LaBrie, M.J. & 671 (NOV) & Melik, E. & 356 (MAY) & & 332 (MAY) \\
\hline Josephson, C.B. & 512 (JULY) & Laforce, R. & $95(\mathrm{JAN})$ & Melik, E.B. & 356 (MAY) & Perassolo, M. & 593 (SEPT) \\
\hline Jung, $S$. & $515(J U L Y)$ & Landry P-A. & $599(\mathrm{SEPT})$ & Melnyk, P. & $599(\mathrm{SEPT})$ & Perez, J. & 365 (MAY) \\
\hline Jurasek, L. & 187 (MAR) & Lasjaunias, P. & 757 (NOV) & Menassa-Moussa, $\mathbf{L}$. & 507 (JULY) & Perez-Larraya, J.G. & 527 (JULY) \\
\hline Juurlink, B.H.J. & $612($ SEPT $)$ & Lau, H.K. & $60(\mathrm{JAN})$ & Merkow, M.B. & 14 (JAN) & Perry, J.R. & 373 (MAY) \\
\hline Kachur, E. & $196(\mathrm{MAR})$ & Laureno, R. & 311 (MAY) & Metz, L.M. & 213 (MAR) & & 654 (SEPT) \\
\hline Kamal, A.K. & 181 (MAR) & Laxmi, V. & 725 (NOV) & Michaud, J. & 751 (NOV) & Phillips, L.A. & 605 (SEPT) \\
\hline Kang, S.Y. & 515 (JULY) & Leblanc, R. & 658 (SEPT) & Miled, A. & 521 (JULY) & Phillips, S. & 731 (NOV) \\
\hline Kaplan, Y. & 575 (SEPT) & Lee, M.J. & 515 (JULY) & Milot, G. & 95 (JAN) & Pierallini, A. & 740 (NOV) \\
\hline Kaprealian, $T$. & 623 (SEPT) & Lee, P.E. & 735 (NOV) & Miropolsky, V. & 745 (NOV) & Pierelli, F. & 740 (NOV) \\
\hline Karaer, $\mathbf{H}$. & 575 (SEPT) & Lee, S-U. & 443 (JULY) & Mitha, A.P. & 776 (NOV) & Pittman, Q.J. & 679 (NOV) \\
\hline Karakas. P. & 356 (MAY) & Lee, T.C. & 373 (MAY) & Mittino, D. & 393 (MAY) & Polat, S. & 356 (MAY) \\
\hline Karam, Y. & $109(\mathrm{JAN})$ & Lemire, E.G. & $105(\mathrm{JAN})$ & Miyajima, $\mathrm{M}$. & 349 (MAY) & Poliquin-Lasnier, L. & 769 (NOV) \\
\hline
\end{tabular}




\section{Author Index to Volume 36 - 2009}

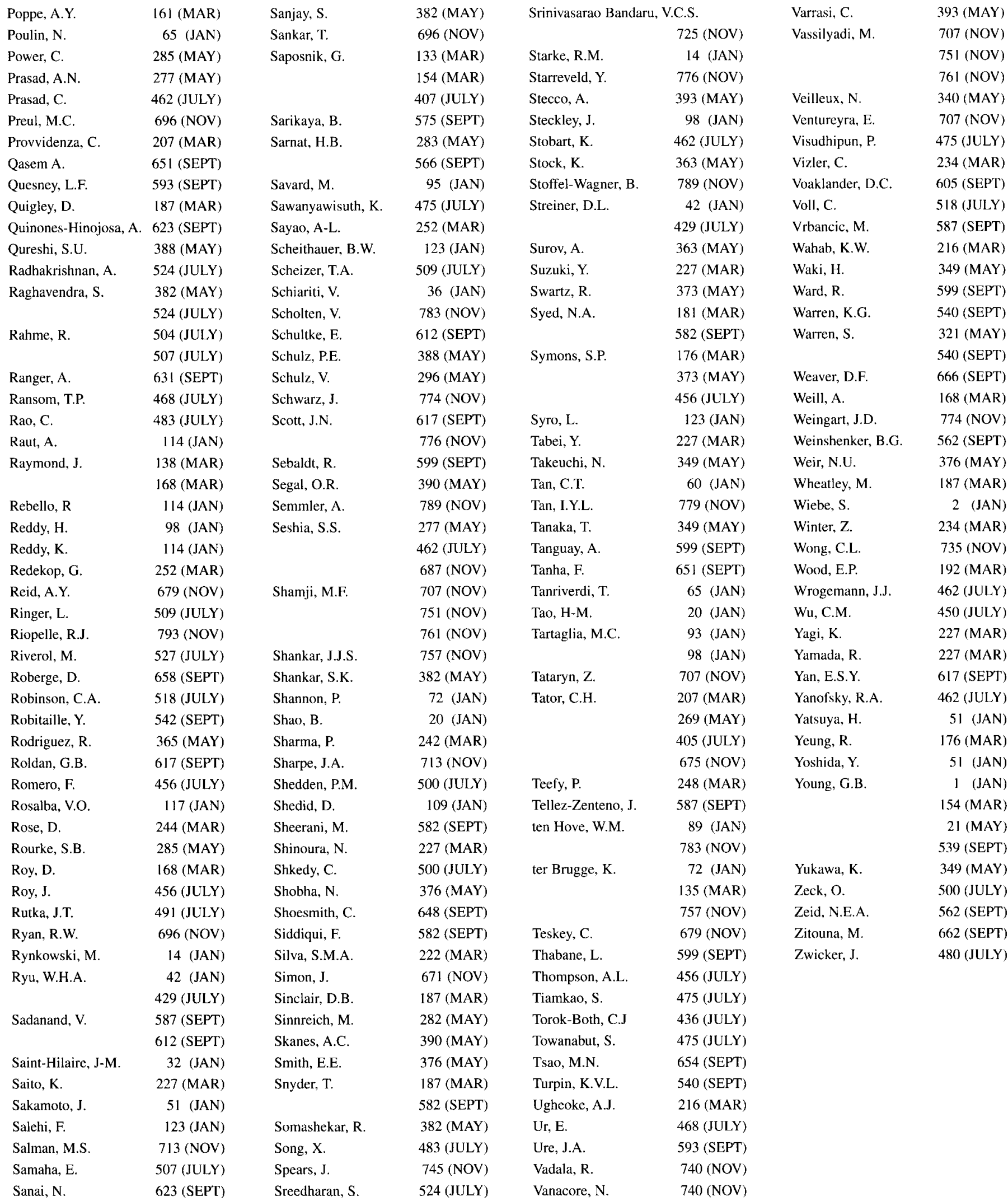

